\title{
Il costo di una neutropenia febbrile
}

\author{
C. Lucioni ${ }^{*}$, L. Crippa $^{\circ}$, O. Leoncini ${ }^{\wedge}$
}

G. Martinelli , S. Mazzi*, A. Santoro ${ }^{\wedge}$

\section{RICERCA}

ORIGINALE

\begin{abstract}
Febrile neutropenia $(\mathrm{FN})$ is one of the most common and severe complications of chemotherapy, particularly when used for the treatment of hematological malignancies and bone marrow transplantation. Estimating its cost for the National Health service (NHS) is difficult, in that the cost of FN is included in the overall reimbursement, which is based on the main DRG declared at discharge.

Aim of the present survey, conducted on the patient population treated in two oncology centers in Milan in the years 2001-2002, was to evaluate the most frequent DRGs associated with neutropenia, with the objective to estimate the mean cost per patient hospitalized with FN.

As expected, oncology patients with NF are spread through a wide range of DRGs, but the mean cost per patient resulted comparable in the two considered hospitals ( $~ 9500$ euro), as well as the overall distribution among DRGs.

The single most frequent DRG was 398 (diseases of reticuloendothelial and immune systems, with complications) which appears to be appropriate, being the closest to the clinical definition of FN available.
\end{abstract}

Keywords: febrile neutropenia, chemotherapy, DRG

Farmeconomia e percorsi terapeutici 2005; 6 (1): 21-24

\section{INTRODUZIONE}

La neutropenia rappresenta il maggiore effetto collaterale della chemioterapia, in particolare quando questa è associata al trattamento dei tumori ematologici e al trapianto di midollo osseo -interventi che richiedono regimi chemioterapici ad alta intensità. Alla severità e alla durata della neutropenia si correla direttamente il rischio di infezione e di conseguente decesso per sepsi. Poiché la febbre può essere la prima e unica manifestazione d'infezione, la prassi clinica prevede che ai pazienti in stato febbrile in un contesto neutropenico siano somministrati antibiotici a largo spettro, normalmente in ricovero ospedaliero, fino a che la febbre sia scomparsa e il conteggio dei neutrofili si sia ripreso.

Valutare il costo di un caso di neutropenia febbrile (NF) pone particolari difficoltà. Infatti, tralasciando l'eventualità di istituire una rilevazione analitica ad hoc, non è possibile utilizzare le tariffe ospedaliere come proxy del costo reale; e questo perchè o la NF si manifesta in pazienti che sono già in corso di assistenza sotto un altro DRG (tipicamente per interventi antitumorali) oppure perché assieme alla NF vengono riscontrati altri sintomi - in tal caso spesso gli agenti patogeni vengono identificati ed è sulla base di questi che il DRG viene assegnato.
Tenendo conto di queste considerazioni, il presente lavoro si propone più semplicemente di verificare a quali tariffe di rimborso ospedaliero viene associato in generale un caso di NF, con l'obiettivo finale di stimare il costo medio di ricovero di un paziente che abbia avuto (anche) un episodio di NF. La prospettiva di valutazione adottata è quella del Servizio Sanitario Nazionale.

\section{MATERIALIE METODI}

Sono stati contattati i responsabili dei reparti di Oncologia Medica ed Ematologia di due Centri, entrambi ubicati a Milano: 1'Istituto Clinico Humanitas (ICH) e l'Istituto Europeo di Oncologia (IEO). Con loro si è convenuto che i casi di pazienti colpiti da NF da selezionare per la ricerca andassero individuati col criterio che nella diagnosi principale o nelle cinque secondarie indicate sulle rispettive SDO fossero presenti:

- il codice ICD-9: 780.6 (FUO, febbre di origine sconosciuta)

- il codice ICD-9: 288.0 (agranulocitosi) simultaneamente al codice 780.6

- un codice ICD-9 compreso tra 001 e 139.8 (estremi del gruppo delle infezioni), assumendo che un'infezione accertata in una
* Wolters Kluwer Health, ADIS International, Milano

- Amgen Italia

^ Istituto Clinico

Humanitas,

Dipartimento di Scienze

Onco-ematologiche

Istituto Europeo di Oncologia, Divisione Onco-ematologica 
Tabella I dei pazienti con neutropenia febbrile $\left(N^{\circ}\right)$
Distribuzione per ICD-9

\begin{tabular}{|c|c|c|c|c|c|c|c|c|c|}
\hline \multirow{2}{*}{ Ricovero } & \multicolumn{4}{|c|}{ Istituto Clinico Humanitas } & \multicolumn{4}{|c|}{ Istituto Europeo di Oncologia } & \multirow{2}{*}{ TOTALE } \\
\hline & FUO ${ }^{(1)}$ & Agranul. & (2) Infezioni (3) & totale & FUO (1) & Agranul. & fezioni ${ }^{(3)}$ & totale & \\
\hline Ordinario & 76 & 88 & 51 & 215 & 16 & 14 & 15 & 45 & 260 \\
\hline D-H & 11 & 0 & 8 & 19 & 4 & 3 & 13 & 20 & 39 \\
\hline Totale & 87 & 88 & 59 & 234 & 20 & 17 & 28 & 65 & 299 \\
\hline
\end{tabular}

situazione di assistenza oncologica fosse generalmente correlata a NF.

(1) Febbre di origine sconosciuta. Casi con ICD-9 780.6 nelle diagnosi (principale o secondarie)

(2) Agranulocitosi con febbre. Casi con ICD-9 288.0 e 780.6 nelle diagnosi (principale o secondarie)

(3) Infezioni

diagnosticate. Casi con ICD-9 da 001 a 139.8

nelle diagnosi

(principale o

secondarie)

(4) Tariffe della Regione Lombardia (DGR n. 941 del 03/08/00)
Ai fini dell'estrazione sono stati considerati tutti i pazienti ricoverati nel 2001 (ICH) o nel 2002 (IEO). Dai database dei due Centri sono stati ripresi diversi dati relativi ai pazienti selezionati - in particolare il DRG che era stato assegnato al loro ricovero.

Per entrambi i Centri i DRG sono stati valorizzati con le tariffe della Regione Lombardia, anno 2000 (DGR VII/941, 03/08/00). In particolare, per sintetizzare le quattro tariffe previste per il DRG 481 (Trapianto di midollo osseo) è stata adottata conservativamente la media delle due più basse. Anche se i ricoveri censiti erano sia in degenza ordinaria che in day hospital, agli effetti del calcolo dei costi sono stati considerati solo i primi, data la loro frequenza preponderante.

\section{RISULTATI}

Nella Tabella I è indicata la conformazione del campione secondo gli ICD-9 adottati come criterio di selezione (a titolo di completezza sono riportati anche i ricoveri in day hospital).

Sempre per completezza di informazione, la Tabella II riporta i DRG sotto i quali sono raggruppati i casi di day-hospital scartati. Si può notare che tali casi presentano la massima frequenza nei ricoveri per chemioterapia.

Le Tabelle III e IV, infine, riportano i risultati delle valorizzazioni, rispettivamente per il Centro ICH e per il Centro IEO. I DRG vi sono elencati in ordine decrescente di frequenza dei casi totali. Ricordiamo che qui sono stati considerati solo i casi di ricoveri in degenza ordinaria.

Il costo medio ponderato di un ricovero in cui il paziente è anche colpito da NF è evidenziato in fondo a ciascuna tabella: 9.805 euro presso l'ICH e 9.259 euro presso l'IEO.

In particolare, tali dati sono analizzati nelle tre componenti costituite dagli altrettanti costi medi parziali che fanno capo alla tripartizione degli ICD-9 adottati come criterio di selezione.

\section{DISCUSSIONE E CONCLUSIONI}

Sia pure con dei limiti (anzitutto la numerosità relativamente bassa delle osservazioni e la provenienza dei dati concentrata in due sole fonti per tanti versi affini) questa indagine conferma che $\mathrm{i}$ casi di pazienti in oncologia colpiti da NF non fanno capo a un solo (o solo alcuni) DRG che potrebbero essere specifici della NF ma, al contrario, sono dispersi in un' area più o meno vasta di DRG loro attribuiti.

Non solo. Anche laddove i casi sono più concentrati, i DRG indicano tipologie di assi-

\begin{tabular}{|c|c|c|c|c|c|c|c|c|c|}
\hline \multirow{2}{*}{ Descrizione } & \multicolumn{4}{|c|}{ Istituto Clinico Humanitas } & \multicolumn{4}{|c|}{ Istituto Europeo di Oncologia } & \multirow{2}{*}{ TOT. } \\
\hline & FUO ${ }^{1}$ & (1) Agranı & zzio & otale & FUO & ans & ezio & otale & \\
\hline 410 Chemioterapia senza dia. second. di leucemia acuta & 3 & - & 4 & 7 & 1 & - & 5 & 6 & 13 \\
\hline 403 Linfoma e leucemia non acuta con CC & - & - & - & - & 1 & 1 & 3 & 5 & 5 \\
\hline 395 Anomalie dei globuli rossi età $>17$ & 4 & - & - & 4 & - & - & - & - & 4 \\
\hline 419 Febbre di origine sconosciuta età $>17$ con CC & 2 & - & - & 2 & 1 & - & - & 1 & 3 \\
\hline 466 Ricoveri succ. senza anamnesi T.M. come dia. second. & - & - & - & - & 1 & - & 2 & 3 & 3 \\
\hline 467 Altri fattori che influenzano lo stato di salute & - & - & 2 & 2 & - & - & 1 & 1 & 3 \\
\hline 398 Disturbi sist. reticoloendot. e immunit. con CC & - & - & 1 & 1 & - & 1 & - & 1 & 2 \\
\hline 145 Altre dia. app. circolatorio senza CC & - & - & - & - & - & - & 1 & 1 & 1 \\
\hline 205 Mal. fegato escl. T.M., cirrosi, epat. alcool. con CC & - & - & 1 & 1 & - & - & - & - & 1 \\
\hline 420 Febbre di origine sconosciuta età $>17$ senza CC & 1 & - & - & 1 & - & - & - & - & 1 \\
\hline 423 Altre dia. infettive e parassitarie & - & - & - & - & - & - & 1 & 1 & 1 \\
\hline 473 Leucemia acuta senza int. chir. maggiori età $>17$ & - & - & - & - & - & 1 & - & 1 & 1 \\
\hline 492 Chemioterapia con dia. second. di leucemia acuta & 1 & - & - & 1 & - & - & - & - & 1 \\
\hline Totale & 11 & 0 & 8 & 19 & 4 & 3 & 13 & 20 & 39 \\
\hline
\end{tabular}

Tabella II

Distribuzione per DRG dei pazienti in D-H (No) 
stenza ospedaliera più comprensive di quella che sarebbe strettamente specifica al trattamento della NF.

Di fatto, dunque, se si utilizzano le tariffe ospedaliere rimborsate, più che valutare il costo di un episodio di NF è possibile vedere in concomitanza a quali altri trattamenti e costi ospedalieri tale episodio si verifica.

Nel presente lavoro tali costi sono stati ottenuti valorizzando i diversi DRG attribuiti ai pazienti, ricoverati presso l'uno o l'altro di due Centri situati nell'area milanese, che avevano avuto anche un episodio di NF. Pesando i valori tariffari con le relative frequenze di attribuzione si sono ottenuti due dati di costo medio, $9.805 \mathrm{e}$ 9.259 euro, che appaiono confrontabili come ordine di grandezza. Anche la distribuzione dei DRG secondo la frequenza complessiva dei casi presso l'uno e l'altro Centro presenta qualche interessante elemento di affinità.

Tuttavia, l'analisi del dato unico di costo medio nelle sue tre componenti (9.557 euro nei casi di FUO, 12.106 euro nei casi di agranulocitosi, 6.204 euro nei casi di infezione

\begin{tabular}{|c|c|c|c|c|c|c|c|}
\hline DRG & Descrizione & $\begin{array}{c}\text { Tariffa }{ }^{(4)} \\
\text { (euro) }\end{array}$ & $\begin{array}{l}\text { FUO }{ }^{(1)} \\
\left(N^{\circ} \text { casi) }\right.\end{array}$ & $\begin{array}{c}\text { Agranul. }{ }^{(2)} \\
\left(\mathrm{N}^{\circ} \text { casi) }\right.\end{array}$ & $\begin{array}{c}\text { Infezioni }{ }^{(3)} \\
\left(\mathrm{N}^{\circ} \text { casi) }\right.\end{array}$ & $\begin{array}{c}\text { Totale } \\
\text { ( } \mathbf{N}^{\circ} \text { casi) }\end{array}$ & $\%$ \\
\hline 398 & Disturbi sist. reticoloendot. e immunit. con CC & 3.166 & 2 & 45 & 7 & 54 & $25,1 \%$ \\
\hline 481 & Trapianto di midollo osseo & 31.762 & 17 & 27 & 4 & 48 & $22,3 \%$ \\
\hline 395 & Anomalie dei globuli rossi età $>17$ & 2.064 & 14 & - & - & 14 & $6,5 \%$ \\
\hline 397 & Disturbi della coagulazione & 3.117 & 3 & 7 & - & 10 & $4,7 \%$ \\
\hline 403 & Linfoma e leucemia non acuta con CC & 4.406 & 3 & - & 7 & 10 & $4,7 \%$ \\
\hline 419 & Febbre di origine sconosciuta età $>17$ con CC & 2.488 & 8 & - & - & 8 & $3,7 \%$ \\
\hline 410 & Chemioterapia senza dia. second. di leucemia acuta & 1.686 & 2 & 1 & 4 & 7 & $3,3 \%$ \\
\hline 416 & Setticemia età $>17$ & 3.986 & 2 & - & 4 & 6 & $2,8 \%$ \\
\hline 89 & Polmonite semplice e pleurite età $>17$ con CC & 3.033 & 4 & 1 & - & 5 & $2,3 \%$ \\
\hline 79 & Infez. e infiamm. respiratorie età > 17 con CC & 4.585 & 2 & 1 & 1 & 4 & $1,9 \%$ \\
\hline 203 & T.M.apparato epatobiliare o pancreas & 3.183 & 1 & - & 3 & 4 & $1,9 \%$ \\
\hline 423 & Altre dia. infettive e parassitarie & 4.183 & - & - & 4 & 4 & $1,9 \%$ \\
\hline 205 & Mal. fegato escl. T.M., cirrosi, epat. alcool. con CC & 3.207 & - & 2 & 1 & 3 & $1,4 \%$ \\
\hline 404 & Leucemia acuta senza int. chir. maggiori età $<18$ & 1.945 & 3 & - & - & 3 & $1,4 \%$ \\
\hline 420 & Febbre di origine sconosciuta età $>17$ senza CC & 1.705 & 3 & - & - & 3 & $1,4 \%$ \\
\hline 473 & Leucemia acuta senza int. chir. maggiori età $>17$ & 10.594 & 3 & - & - & 3 & $1,4 \%$ \\
\hline 20 & Infez. sist. nervoso escl. meningite virale & 6.171 & - & - & 2 & 2 & $0,9 \%$ \\
\hline 188 & Altre dia. app. digerente età $>17$ con CC & 2.611 & - & - & 2 & 2 & $0,9 \%$ \\
\hline 316 & Insufficienza renale & 3.377 & 1 & - & 1 & 2 & $0,9 \%$ \\
\hline 401 & Linfoma e leucemia non acuta con altri con int. con CC & 7.236 & - & 1 & 1 & 2 & $0,9 \%$ \\
\hline 402 & Linfoma e leucemia non acuta con altri con int. senza CC & 2.235 & 2 & - & - & 2 & $0,9 \%$ \\
\hline 492 & Chemioterapia con dia. second. di leucemia acuta & 8.565 & - & 2 & - & 2 & $0,9 \%$ \\
\hline 1 & Craniotomia età $>17$ escl. per traumatismo & 9.954 & - & - & 1 & 1 & $0,5 \%$ \\
\hline 18 & Mal. nervi cranici e periferici con CC & 2.401 & - & - & 1 & 1 & $0,5 \%$ \\
\hline 85 & Versamento pleurico con CC & 3.134 & 1 & - & - & 1 & $0,5 \%$ \\
\hline 87 & Edema polmonare e insuff. respiratoria & 3.560 & 1 & - & - & 1 & $0,5 \%$ \\
\hline 94 & Pneumotorace con CC & 3.345 & - & 1 & - & 1 & $0,5 \%$ \\
\hline 108 & Altri int. cardiotoracici & 16.455 & - & - & 1 & 1 & $0,5 \%$ \\
\hline 143 & Dolore toracico & 1.352 & - & - & 1 & 1 & $0,5 \%$ \\
\hline 172 & T.M. app. digerente con CC & 3.401 & 1 & - & - & 1 & $0,5 \%$ \\
\hline 199 & Proc. diagnost. epatobiliari per T.M. & 7.198 & - & - & 1 & 1 & $0,5 \%$ \\
\hline 272 & Mal. maggiori della pelle con CC & 2.743 & - & - & 1 & 1 & $0,5 \%$ \\
\hline 320 & Infezioni rene e vie urinarie età $>17$ con CC & 2.568 & 1 & - & - & 1 & $0,5 \%$ \\
\hline 350 & Infiammazioni app. riproduttivo maschile & 1.756 & - & - & 1 & 1 & $0,5 \%$ \\
\hline 394 & Altri int. sugli organi emopoietici & 4.181 & - & - & 1 & 1 & $0,5 \%$ \\
\hline 399 & Disturbi sist. reticoloendot. e immunit. senza CC & 1.764 & 1 & - & - & 1 & $0,5 \%$ \\
\hline 400 & Linfoma e leucemia con int. chir. maggiori & 7.874 & 1 & - & - & 1 & $0,5 \%$ \\
\hline 413 & Altre alterazioni mieloprolif., etc. con CC & 3.492 & - & - & 1 & 1 & $0,5 \%$ \\
\hline \multirow[t]{3}{*}{463} & Segni e sintomi con CC & 1.905 & - & - & 1 & 1 & $0,5 \%$ \\
\hline & totale casi & & 76 & 88 & 51 & 215 & $100,0 \%$ \\
\hline & costo medio (euro) & & 9.557 & 12.106 & 6.204 & 9.805 & \\
\hline
\end{tabular}

\section{Tabella III}

Costo medio di un ricovero ordinario con episodio di neutropenia presso il Centro ICH 


\begin{tabular}{|c|c|c|c|c|c|c|c|}
\hline DRG & Descrizione & $\begin{array}{c}\text { Tariffa }{ }^{(4)} \\
\text { (euro) }\end{array}$ & $\begin{array}{c}\text { FUO }{ }^{(1)} \\
\left(N^{\circ} \text { casi) }\right.\end{array}$ & $\begin{array}{l}\text { Agranul. }{ }^{(2)} \\
\left(\mathrm{N}^{\circ} \text { casi) }\right.\end{array}$ & $\begin{array}{l}\text { Infezioni }{ }^{(3)} \\
\left(N^{\circ} \text { casi) }\right.\end{array}$ & $\begin{array}{c}\text { Totale } \\
\text { ( } \mathrm{N}^{\circ} \text { casi) }\end{array}$ & $\%$ \\
\hline 398 & Disturbi sist. reticoloendot. e immunit. con CC & 3.166 & - & 7 & 2 & 9 & $20,0 \%$ \\
\hline 403 & Linfoma e leucemia non acuta con CC & 4.406 & 2 & 4 & 3 & 9 & $20,0 \%$ \\
\hline 481 & Trapianto di midollo osseo & 31.762 & 5 & 1 & 3 & 9 & $20,0 \%$ \\
\hline 410 & Chemioterapia senza dia. second. di leucemia acuta & 1.686 & 1 & 1 & 3 & 5 & $11,1 \%$ \\
\hline 492 & Chemioterapia con dia. second. di leucemia acuta & 8.565 & 2 & 1 & 1 & 4 & $8,9 \%$ \\
\hline 404 & Leucemia acuta senza int. chir. maggiori età $<18$ & 1.945 & 3 & - & - & 3 & $6,7 \%$ \\
\hline 89 & Polmonite semplice e pleurite età $>17$ con CC & 3.033 & 1 & - & - & 1 & $2,2 \%$ \\
\hline 203 & T.M.apparato epatobiliare o pancreas & 3.183 & 1 & - & - & 1 & $2,2 \%$ \\
\hline 272 & Mal. maggiori della pelle con CC & 2.743 & - & - & 1 & 1 & $2,2 \%$ \\
\hline 419 & Febbre di origine sconosciuta età $>17$ con CC & 2.488 & 1 & - & - & 1 & $2,2 \%$ \\
\hline 421 & Mal. di origine virale età $>17$ & 1.802 & - & - & 1 & 1 & $2,2 \%$ \\
\hline \multirow[t]{3}{*}{465} & Ricoveri succ. con anamnesi T.M. come dia. second. & 888 & - & - & 1 & 1 & $2,2 \%$ \\
\hline & totale casi & & 16 & 14 & 15 & 45 & $100,0 \%$ \\
\hline & costo medio (euro) & & 12.561 & 5.843 & 8.926 & 9.259 & \\
\hline
\end{tabular}

Tabella IV

Costo medio di un ricovero ordinario con episodio di neutropenia presso il Centro IEO

\section{Legenda tabelle}

(1) Febbre di origine sconosciuta. Casi con ICD-9 780.6 nelle diagnosi (principale o secondarie)

(2) Agranulocitosi con febbre. Casi con ICD-9 288.0 e 780.6 nelle diagnosi (principale o secondarie)

(3) Infezioni

diagnosticate. Casi con ICD-9 da 001 a 139.8 nelle diagnosi (principale o secondarie)

(4) Tariffe della Regione Lombardia (DGR n. 941 del 03/08/00) presso l'ICH; e analogamente 12.561, $5.483 \mathrm{e}$ 8.926 euro presso l'IEO) rivela strutture di costo non analoghe. Ciò si spiega in gran parte perchè col DRG 481 (Trapianto di midollo osseo, cioè quello col massimo valore di rimborso) risultano essersi avute soprattutto diagnosi di agranulocitosi presso l'ICH e diagnosi di FUO presso l'IEO.

Peraltro, si può infine osservare che il DRG che verosimilmente si potrebbe ritenere il più vicino agli effettivi casi di ricovero per NF è il 398 (Disturbi del sistema reticoloendoteliale e immunitario con $\mathrm{CC}$ ). Esso risulta il DRG di più frequente utilizzo in entrambe le istituzioni sia in generale per tutti i casi, sia soprattutto nei casi che presentano agranulocitosi con febbre (ICD9288.0 e 780.6). La tariffa di rimborso del DRG 398 , pari a 3.166 euro potrebbe quindi essere rappresentativa di un possibile costo medio di ricovero conseguente a un episodio di NF.

Per ulteriore documentazione abbiamo ricalcolato i costi sostituendo alle tariffe regionali della Lombardia del 2000 quelle aggiornate al 2003 (DGR VII/15325, 28/11/2003). Sono state in- fine utilizzate anche le tariffe nazionali (DM 12/ 03/97), anche se queste ultime non hanno ormai che un mero valore di riferimento dal momento che ogni Regione adotta il proprio sistema tariffario. Nella Tabella V viene riportato un riepilogo comparativo.

Come si può notare, mentre con l'adozione delle nuove tariffe della Regione Lombardia il costo medio di un ricovero per NF salirebbe appena di qualche punto percentuale, con le tariffe nazionali esso risulterebbe superiore di un terzo e oltre.

Per quanto riguarda invece, in particolare, il DRG maggiormente associato ai ricoveri per NF $(398$ - Disturbi del sistema reticoloendoteliale e immunitario con cc), la rispettiva tariffa in Lombardia è rimasta invariata fra il 2000 e il 2003: 3.166 euro, a fronte di quella a livello nazionale che ammonta a un valore quasi doppio (5.964 euro).

Per mancanza di dati non è stato purtroppo possibile, anche se sarebbe stato di grande interesse, confrontare i costi effettivamente sostenuti dagli ospedali per gestire i pazienti neutropenici con i rimborsi ottenuti.

Questo studio è stato reso possibile da un finanziamento messo a disposizione da Amgen Italia SpA

\begin{tabular}{l|ccccc|ccccc}
\hline \multirow{2}{*}{$\begin{array}{c}\text { Reparto di Oncologia } \\
\text { Medica ed Ematologia }\end{array}$} & \multicolumn{4}{c}{ Istituto Clinico Humanitas } & \multicolumn{5}{c}{ Istituto Europeo di Oncologia } \\
\cline { 2 - 11 } & FUO (1) & Agranul. (2) & Infezioni (3) & totale & N.I. & FUO (1) & Agranul. (2) Infezioni (3) & totale & N.I. \\
\hline con tariffe Lombardia 2000 & 9.557 & 12.106 & 6.204 & 9.805 & 100 & 12.561 & 5.843 & 8.926 & 9.259 & 100 \\
con tariffe Lombardia 2003 & 9.673 & 12.351 & 6.334 & 9.977 & 102 & 13.000 & 6.111 & 9.280 & 9.617 & 104 \\
con tariffe nazionali & 12.938 & 16.635 & 7.858 & 13.246 & 135 & 16.083 & 8.604 & 11.767 & 12.318 & 133 \\
\hline
\end{tabular}

Tabella V

Riepilogo comparativo del costo medio di un ricovero ordinario con episodio di NF 